\title{
Experimental demonstration of counterfactual quantum communication
}

\author{
Yang Liu ${ }^{\dagger}$, Lei $\mathrm{Ju}^{\dagger}$, Xiao-Lei Liang, \\ Shi-Biao Tang, Guo-Liang Shen Tu, Lei Zhou, Cheng-Zhi Peng, \\ Kai Chen, Teng-Yun Chen*, Zeng-Bing Chen,and Jian-Wei Pan* \\ Hefei National Laboratory for Physical Sciences at Microscale \\ and Department of Modern Physics, \\ University of Science and Technology of China, Hefei, Anhui 230026, China
}

†These authors contributed equally to this work.
*To whom correspondence should be addressed. E-mail: tychen@ustc.edu.cn,pan@ustc.edu.cn

Based on principle of quantum mechanics, quantum cryptography provides an intriguing way to establish secret keys between remote parties, generally relying on actual transmission of signal particles. Surprisingly, an even more striking method is recently proposed by Noh (1) named as 'counterfactual quantum cryptography' enabling key distribution, in which particles carrying secret information are seemly not being transmitted through quantum channel. We experimentally give here a faithful implementation by following the scheme with an on-table realization. Furthermore, we report an illustration on a $1 \mathrm{~km}$ fiber operating at telecom wavelength to verify its feasibility for extending to long distance. For both cases, high 


\section{visibilities of better than $98 \%$ are maintained with active stabiliza- tion of interferometers, while a quantum bit error rate around $5.5 \%$ is attained after $1 \mathrm{~km}$ channel.}

The principle of quantum mechanics provides exciting opportunities across a number of applications which offer high-performance and superiority over classical ways, among which quantum key distribution appears as the first practical application with provable unconditional security between communication parties. Since proposal of BB84 protocol (2) for quantum key distribution, there have spurred a flurry of activity in both theoretical developments and successful experimental demonstrations of quantum cryptography systems ranging from a two-party communication up to a network (2-21). Particularly, recent advances have covered high speed and long distance demonstrations under imperfect devices, by utilizing decoy state method $(6,8,9,12-16,19-21)$. In order to produce identical secure keys between two remote communication parties, key distribution process generally require actual transmission of signal particles through a quantum channel, before detections and data post-processing.

In conformity with quantum mechanics, there are many fundamental quantum phenomena that lead to intriguing physical effects (22-26). Striking applications are consequently proposed like interaction-free measurement (22), counterfactual quantum computation (23), in which the presence of a nontransmitting object is ascertained seemingly without interacting with it, or outcomes of computations can sometimes be inferred without running of a computer, as successfully demonstrated in (24) and (25), respectively. Following this route, Noh has presented an engaging method to do key distribution, named as 'counterfactual quantum cryptography' (1). This engrossing proposal gives not only a conceptually new approach to do secure communication by use of counterfactual characterization in quantum phenomena, but also it promises potential security advantage 
for communication. In the scheme, quantum signals producing secure final keys are not actually transmitted. Recently an unconditional security proof is provided in the case of ideal case by utilizing perfect single-photon source and with well-controlled detectors' efficiencies etc (27). An attempt is made in (28), in which a modified realization of the proposal is presented. The original scheme is, however, not be justified by the demonstration, whose equivalence and security need additionally thorough analysis. Fascinatingly, the counterfactual method itself provides an employment of quantum principles for applicable task, and motivates us a more novel conceptually thinking for fundamental quantum mechanics.

We present here experimentally a faithful implementation of the counterfactual quantum communication (CQC) operating at telecom wavelength. we have first set up the experiment on a table, and consequently extended it to a $1 \mathrm{~km}$ fiber to check feasibility for long-distance realization. Active feed-back control technology is developed for realtime stabilization of Michelson-type interferometer utilized in the scheme, to guarantee favorable condition for successful operation of quantum communication. In addition, tailored optical and controlling electronics designs are made, which enables to attain a high visibility of better than $98 \%$ for interferometer through $1 \mathrm{~km}$ long fiber.

A schematic of the experimental setup is illustrated in Fig. 1. Two distributed feedback laser diodes (LDs) with pulse width about $1 \mathrm{~ns}$ for generating horizontal and vertical polarizations are combined with a polarization beam splitter (PBS) to act as signal light. The center wavelengths of the two LDs were carefully tuned to be consistent within a range of $0.02 \mathrm{~nm}$. Random number will be supplied to enable Alice to generate a sequence of pulses with random polarization $\mid H>$ or $\mid V>$. An attenuator is used to attenuate the pulse to single photon level at Alice's output port. As depicted by the protocol, the signal pulse is split by a beam splitter (BS), and then travels through arm $a$ or arm $b$ for 
transmission. A polarization controller (PC) is inserted for compensating possible polarization drift of fiber channel. In the protocol, Bob will randomly choose one of the two polarizations representing his bit value. The optical path $b$ of the single-photon pulse will be blocked if the polarization of the pulse is identical to his polarization. For achieving this aim, a tailored PBS is used with the reflecting port connected with a jumper, which is about $20 \mathrm{~m}$ long, enough for accumulating time needed for changing status of optical switch (SW). This design allows to set a delay for $\mathrm{H}$ and $\mathrm{V}$ pulse: horizontal light will pass the PBS directly, while vertical light will reflect to the delay line, and then transmit back to another output port of PBS before going through SW. Bob can therefore control accurately switch timing to guide signal photon to be directly detected by his polarization sensitive detector, or to be rotated and reflected by Faraday mirror (FM) and to return back to BS. In our experiment, polarization sensitive detecting is accomplished by a PBS and two single photon detectors. Therefore through accurate control of the switch timing, Bob can determine effectively whether switch the polarization state to the detector D3 or not.

If Bob chooses a different polarization from Alice, the pulse will be reflected by Bob and combined again at BS. In this case, the photonic pulse will go toward detector D2 due to interference effect if there is a phase difference of $\pi$ radians between the two paths $a$ and $b$. An attenuator is added to arm $a$ to balance the optical loss between two arms. To compensate the phase drift in the fiber, and to be sure that all light after interference will go to detector $D 2$, a fast fiber stretcher (FS) is used for compensating fast phase variation in the two arms. Moreover, an optical delay line (OD) is employed by Alice to adjust the path difference between arm $a$ and arm $b$ of the whole system with an precision of less than $0.1 \mathrm{~mm}$. In order to obtain good visibility in the interferometer, feedback control is further developed by using a laser with different wavelength of $1570 \mathrm{~nm}$ and controlling 
electronics. The feedback laser and the signal laser is combined by a dense wavelength division multiplexer (DWDM) before entering BS.

If their choices are same, the interferometer will be destroyed, since Bob chooses to detect rather than let it go through, either $D 1, D 2$ of Alice or $D 3$ of Bob will detect a pulse signal. We save only events for $D 1$ clicks, and kept all other events as a monitoring of Eve's intervention. For $D 1$ 's clicks, only the events for which $D 1$ detects a correct final polarization will be kept by Alice and no information is revealed, otherwise measurements results are announced. For $D 2$ or D3's clicks, both detected polarization and initial polarization states are released for detection of possible eavesdropping. Accord to the scheme, the initial quantum state after the BS can be rephrased as one of the following two orthogonal states, $\left|\phi_{0}>=\sqrt{T}\right| 0>_{a}\left|H>_{b}+i \sqrt{R}\right| H>_{a}\left|0>_{b},\right| \phi_{1}>=\sqrt{T}\left|0>_{a}\right| V>_{b}$ $+i \sqrt{R}\left|V>_{a}\right| 0>_{b}$. Here $R$ and $T$ are the reflectivity and transmissivity rate of the BS, respectively. In our case $R$ and $T$ are $50 \% / 50 \%$. The $a$ and $b$ denote the path toward Alice's Faraday mirror, and the path toward Bob site, respectively. We use $\mid 0>_{a}$ and $\mid 0>_{b}$ to represent the vacuum state in the modes $a$ and $b$, respectively. As for the events for which $D 1$ detects a correct final polarization, the initial states will collapse into two states of $\left|H>_{a}\right| 0>_{b}$ or $\left|V>_{a}\right| 0>_{b}$. In fact, Bob will extract secure keys with Alice from nondetection events.

In the experiment we use $1550 \mathrm{~nm}$ DFB laser as signal source that is actively modulated into about $1 \mathrm{~ns}$ pulse width. The intensity of laser was attenuated to $0.1 \sim 1$ photon/pulse on average at the output of Alice's side. The total loss of the $\operatorname{arm} b$ was measured to be around $10.5 \mathrm{~dB}$ when photon goes through and returns back after Bob's FM for case of an on-table test. The system works at a repetition rate of $100 \mathrm{KHz}$. Currently this is mainly limited by the performance of feedback loop. In addition, response time of the fiber switch of about $100 \mathrm{~ns}$, and switching time of about $300 \mathrm{~ns}$ are needed to choose the 
polarization of each pulse.

An active feedback control system is developed for phase stabilization. Using a DWDM, we couple the signal light, and the 1570nm reference light of a DFB CW laser, before their entering at the BS. When the system works in feedback mode, Bob always selects to reflect the reference light by choosing the optical switch to the FM. The reference light goes through the interferometer via the same path of signal light, and goes back through DWDM to a different output of optical circulator. A highly sensitive APD-diode D0 is used to detect the intensity of the output of interferometer. Thus, the reference light goes through the same interferometer as the signal light, and experiences the same path-difference. However the phase differences are different due to different wavelengths of signal and reference light. Following a similar analysis shown in (31), we can illustrate the phase difference with $n \triangle L=\lambda_{r}\left(m_{r}+\varphi_{r} / 2 \pi\right)=\lambda_{s}\left(m_{s}+\varphi_{s} / 2 \pi\right)$. Here $n$ is the refractive index, subscripts $r, s$ refer to reference and signal light, respectively. $\triangle L$ is path difference. The relative phase difference $\varphi_{r}, \varphi_{s}$ have a relation with its wavelength $\lambda$ for a fixed setup. The $m$ is integer, indicating phase difference being of integral multiple of its wavelength. The intensity in the output of interferometer can be given by $I=I_{0} \cdot \cos ^{2}\left(\frac{k \triangle L}{2}\right)$ where $k$ is wave number. By stabilizing the output intensity of reference to a settled level, one can thus fix the path difference for signal light. Experimentally, a homemade PI controller from cold-atom experiment is modified and adjusted for experimental need. A voltage amplifier is used to control the fiber stretcher (FS), which can fast change the relative length of fiber in path $a$. The PI controller compares the output of APD-diode with a pre-set monitor level. If the output voltage of APD-diode differs from a monitor level, PI circuit will adjust the output voltage for compensating the change. For our $1 \mathrm{~km}$ system, phase change is roughly in a level of $0.1 \sim 0.5 \mathrm{rad} / \mathrm{ms}$. A total range of $20 \mathrm{~V}(-10 \mathrm{~V} \sim+10 \mathrm{~V})$ output with subsequent amplification factor of $10 \sim 40$ 
times enables to make a $200 \pi$ rad adjustment through fiber stretcher. A final interference visibility of better than $98 \%$ is observed for both the case of desktop test and the $1 \mathrm{~km}$ fiber cable application, which enables successful operations of feedback controlling system. Continuous running of about an hour for desktop test, and $900 \mathrm{~s}$ for $1 \mathrm{~km}$ application have shown stable and reliable performances.

An FPGA electronics board is developed for controlling signal laser, detectors and fiber switch. The timing is about $1 \mu s$ for signal pulse generation and detection, and 9 $\mu s$ feedback time for proportional-integral (PI) circuit, within a $10 \mu s$ duty circle for FPGA. We use one $1570 \mathrm{~nm}$ DFB laser as reference light. We have firstly implemented the scheme in case of on-table test. With PI feedback, a fringe visibility of better than $98 \%$ could be maintained. A raw key of 185651 bits is generated within a 3617s running (amounting to $51.3 \mathrm{bits} / \mathrm{s}$ ), with an average quantum bit error rate (QBER) of $6.8 \%$ when the average photon number is 0.5 photon/pulse. If we change the average photon number to be 0.05 photon/pulse, the raw key of 7891 bits will be produced with QBER of $4.2 \%$ on average. To test feasibility of CQC, we have extended the scheme with a 1 km fiber cable connecting Alice and Bob's sites, which will cause about $1 \mathrm{~dB}$ for channel loss. A fringe visibility of about $98 \%$ is managed to be fulfilled as well for $1 \mathrm{~km}$ fiber, which allows generation of secure keys. Without any cover for fibers on experimental desktop, a raw key of about 126 bits/s is obtained with QBER of about $5.8 \%$ for $900 \mathrm{~s}$ continuous running, when the average photon number is chosen to be 1.0 photon/pulse. If the average photon number is reduced to 0.5 photon/pulse, the measured QBER is about $5.5 \%$ while raw keys is decreased to be around 94 bits/s due to longer feedback time. See Table 1 for performance details. We can see that for $1 \mathrm{~km}$ fiber realization, one can obtain considerable raw key rates of 126 bits/s and good performance, with a quite low error rate of $0.6 \%$ through $D 3$ for channel monitoring, when using average photon 
number of 1.0 .

In order to have a more comprehensive understanding for running of the experiment, we present here a circumstantial analysis for experimental imperfections. Consider we have two groups of data, one group that Alice and Bob choose the same random numbers, and another group that they choose different random numbers. The group with the same number will create a raw key if D1 is detected with correct basis, which will be fulfilled if the polarization is not disturbed. Another group will create a possible wrong key if D1 is detected with correct basis, which will contribute to an error. Here we take the case of $1 \mathrm{~km}$ experiment with the mean photon number of 0.5 for an example to analyze. We attribute all the errors coming mainly from three parts. The first part is from dark counts and after-pulses of the detectors. The detectors we used have a dark count possibility of each gate of about $10^{-5}$. With $100 \mathrm{kHz}$ working frequency, about 1 dark count is created by detector, which will therefore contribute to about $0.7 \%$ error rate, as a total counts of $D 1$ is about 70 counts per second in this case. The after pulse will lead to about $0.5 \%$ error rate due to a $1 \%$ probability of after pulse for detectors. The second part is from finite extinct ratio of fiber switch. We have tested the static extinction ratio of the fiber switch to be $20 \mathrm{~dB}$, with a slight lower of about $17 \mathrm{~dB}$ with regarding to the dynamic extinction ratio, which amounts approximately to $1 \% \sim 2 \%$ error rate. The third one is coming from imperfection of the optical alignment and the finite visibility of feedback system, which will still lead clicks for $D 1$ even if Alice and Bob have different choices. When Alice and Bob choose different random numbers, a visibility like $98 \%$ is supposed to be achieved. This means that $1 \%$ of the pulses are detected by D1 and that the other $99 \%$ are detected by D2. The error rate can thus be estimated as $0.01 /(0.01+0.25)=3.8 \%$, by considering the fact that only $1 / 4$ of the total pulses will be detected by D1 when Alice and Bob choose same random number. All three parts therefore would conduct to 
a QBER of around 6\% as confirmed by actual measured experimental data.

A preliminary security analysis is given in (1) by showing a new type of noncloning principle for orthogonal states: if reduced density matrices of an available subsystem are nonorthogonal and if the other subsystem is not allowed access, it is impossible to distinguish two orthogonal quantum states $\mid \phi_{0}>$ and $\mid \phi_{1}>$. without disturbing them. Moreover the scheme is robust to so-called "photon-number splitting" attack. Since Eve cannot access nondetection process that extracts secret keys (1), these distinctive property provides a security advantage over existing schemes. Consider in a typical noise channel case as analyzed in (27) in which possible upper bound for phase error rate could be less than QBER, our system allows sufficiently to generate secure keys after one-way key distillation process (4) for ideal case of source and detectors.

We have achieved the faithful and complete realization of counterfactual quantum communication, in which process information carriers are seemingly not traveled in the quantum channel. From a desktop test to a setup with $1 \mathrm{~km}$ fiber cable, we have given proof-in-principle demonstrations of CQC. This gives a confirming answer for feasibility of $\mathrm{CQC}$, and one can infer that the mere possibility for signal particles to be transmitted is sufficient to create a secret key. We remark that, to ensure such possibility, partial signal particles still need to randomly travel along quantum channel for detection of possible eavesdropping. One may wonder in the implemented scheme, signals pass through the channel twice and thus suffer losses twice. It should be remarked that the CQC scheme is, in fact, equivalent to the normal BB84 protocol for communication overhead of transmission loss, if one assists with a quantum repeater through DLCZ scheme (29) to teleport single photon signal directly between Alice and Bob. Also one may see that the scheme requires to maintain long-term subwavelength stability of the path difference between two arms of a long-distance interferometer, which can however be overcome by 
utilizing state-of-the-art technology, similar to the one developed in (30) for coherent optical phase transfer over $30 \mathrm{~km}$ fiber. We can see that our implementation is based on currently available technologies, promising a novel conceptually quantum communication system compared with existing systems. Presently the performance of our system is mainly limited by speed of feedback. Consider our feedback system is only a modification of PI used in our cold-atom experiments, significant improvements could be made by exploiting the method developed in (31) for interferometer stabilization, and by employing tailored design with a differential circuit, an auto-reset function and exploiting adjacent channel in $\mathrm{C}$ band of $1550.12 \mathrm{~nm}$. Although technically challenging, our implementation in conjunction with optimizing quality of interference via improved optical design, appears as a viable route toward extendable realization up to long distance fiber.

\section{References and Notes}

1. T.G. Noh, Phys. Rev. Lett. 103, 230501 (2009).

2. C.H. Bennett, G. Brassard, Quantum cryptography: public key distribution and coin tossing, in Proceedings of the IEEE International Conferenceon Computers, Systems and Signal Processing, (Bangalore, India, 1984), pp. 175-179.

3. H.-K. Lo, H.F. Chau, Science 283, 2050 (1999).

4. P.W. Shor, J. Preskill, Phys. Rev. Lett. 85, 441 (2000).

5. N. Gisin, G. Ribordy, W. Tittel, H. Zbinden, Rev. Mod. Phys. 74, 145 (2002).

6. W.Y. Hwang, Phys. Rev. Lett. 91, 057901 (2003).

7. D. Gottesman, H.-K. Lo, N. Lütkenhaus, J. Preskill, Quant. Inf. Comput. 5, 325 $(2004)$ 
8. X.-B. Wang, Phys. Rev. Lett. 94, 230503 (2005).

9. H.-K. Lo, X.-F. Ma, K. Chen, Phys. Rev. Lett. 94, 230504 (2005).

10. C. Elliott et al., Current status of the DARPA Quantum Network, in Quantum Information and Computation III, Donkor, E.J., Pirich, A.R., \& Brandt, H. E. eds., Proc. SPIE 5815, 138-149 (2005).

11. H. Inamori, N. Lütkenhaus, D. Mayers, Eur. Phys. J. D 41, 599 (2007).

12. C.-Z. Peng et al., Phys. Rev. Lett. 98, 010505 (2007).

13. D. Rosenberg et al., Phys. Rev. Lett. 98, 010503 (2007).

14. T. Schmitt-Manderbach et al., Phys. Rev. Lett. 98, 010504 (2007).

15. Z.-L. Yuan, A.W. Sharpe, A.J. Shields, Appl. Phys. Lett. 90, 011118 (2007).

16. M. Peev et al., New J. Phys. 11, 075001 (2009).

17. V. Scarani, H.B. Pasquinucci, N.J. Cerf, M. Dusek, N. Lütkenhaus, M. Peev, Rev. Mod. Phys. 81, 1301(2009).

18. Q. Zhang et al., New J. Phys. 11, 045010 (2009).

19. W. Chen et al., IEEE Photonics. Tech. Letts. 21, 575 (2009).

20. T.-Y. Chen et al., Opt. Exp. 18, 27217 (2010).

21. M. Sasaki et al., Opt. Exp. 19, 10387 (2011).

22. A.C. Elitzur, L. Vaidman, Found. Phys. 23, 987 (1993).

23. G. Mitchison, R. Jozsa, Proc. R. Soc. Lond. A 4571175 (2001). 
24. P. G. Kwiat et al., Phys. Rev. Lett. 83, 725 (1999).

25. O. Hosten, M.T. Rakher, J.T. Barreiro, N.A. Peters, P.G. Kwiat, Nature 439, 949 (2006).

26. V. Parigi, A. Zavatta, M.S. Kim, M. Bellini, Science 317, 1890 (2007).

27. Z.-Q. Yin, H.-W. Li, W. Chen, Z.-F. Han, G.-C. Guo, Phys. Rev. A 82, 042335 (2010).

28. M. Ren, G. Wu, E. Wu, H. Zeng, Laser Phys. 21, 755-760 (2011).

29. L.-M. Duan, M.D. Lukin, J.I. Cirac, P. Zoller, Nature 414, 413 (2001).

30. S.M. Foreman, A.D. Ludlow, M.H.G. de Miranda, J.E. Stalnaker, S.A. Diddams, J. Ye, Phys. Rev. Lett. 99, 153601 (2007).

31. S.-B. Cho, T.-G. Noh, Opt. Exp. 17, 19027 (2009).

32. We acknowledge the financial support from the CAS, the National Fundamental Research Program of China under Grant No.2011CB921300, the National High Technology Research and Development Program (863 Program) of China under Grant No.2009AA01A349, the NNSFC and the Fundamental Research Funds for the Central Universities. The authors are grateful for valuable discussions with Dr. Xian-Min Jin. 


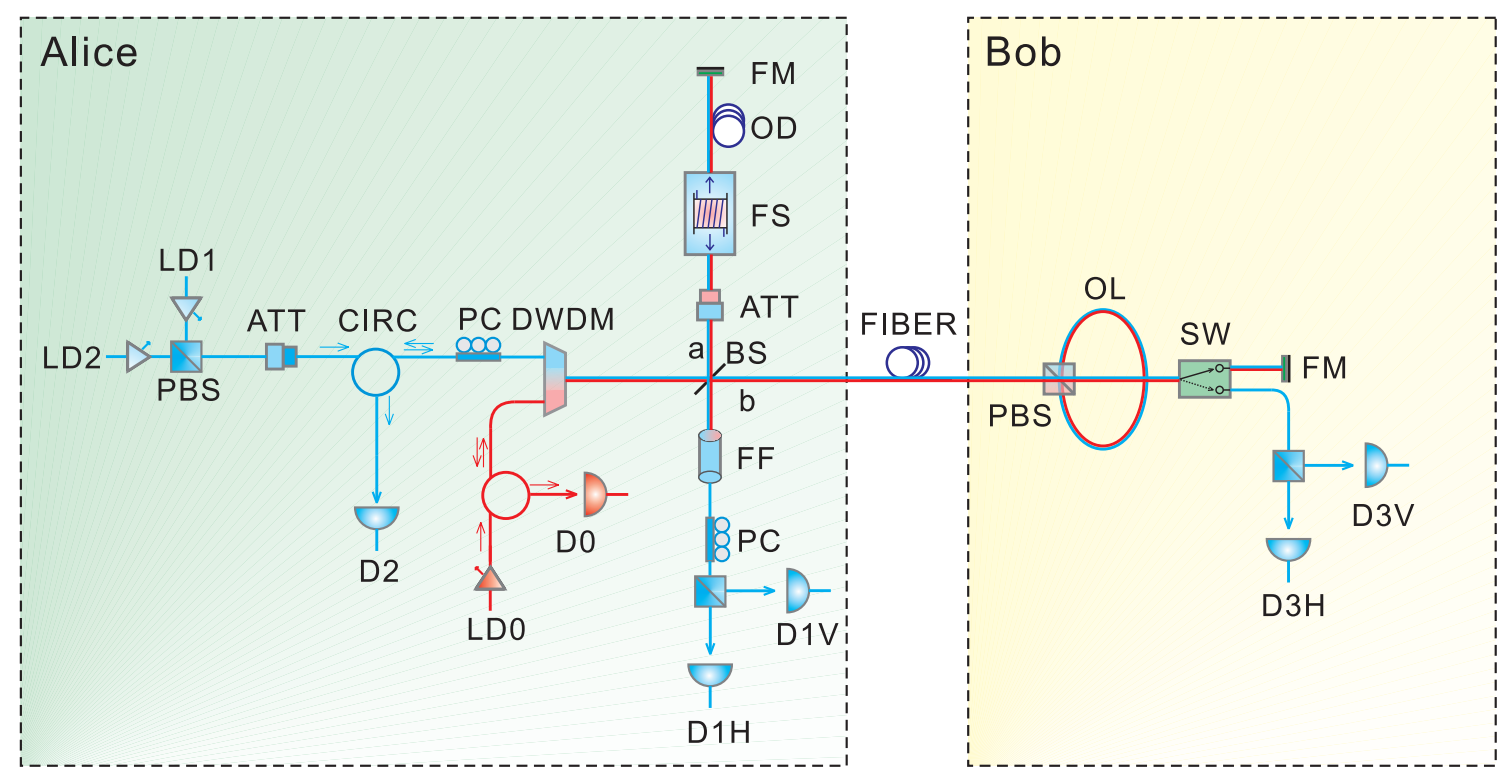

Fig. 1. Schematic setup of fiber based counterfactual quantum cryptography system. A signal photonic pulse is sent from Alice's side before passing through a optical circulator and then splitting by a BS to travel along path $a$ and $b$. Alice chooses randomly one of states of horizontal polarization $\mid H>$ representing "0", and vertical polarization $\mid V>$ representing " 1 ". Depends on Bob's choice coinciding with Alice's choice or not, the two split pulses will be recombined in the BS and detected by detector D2 due to constructive interference, or a split pulse will go through path $b$ and be blocked by detector D3. As a result of latter case, interference is destroyed and D1 will detect a photon will certain probability. Sifted keys will be established by selecting only the events for which $D 1$ alone detects a photon with a correct final polarization state. Note that, when $D 1$ clicks the photon only travels inside Alice's secure zone, and there's no photon travel to Bob's side, which excludes Eve's access for each signal particle of the entire quantum system. LD: laser diode, PBS: Polarization Beam Splitter, ATT: fiber attenuator, CIRC: 
optical circulator, PC: polarization controller, BS: Beam Splitter, FS: Fiber stretcher, DWDM: Dense wavelength division multiplexer, FF: Fiber Filter, OD: Optical delay line, OL: Optical delay, SW: fiber switch, FM: Faraday rotator mirror. D0,D1,D2,D3: detectors. D1H, D1V are short notions for detector D1 of detecting horizontal and vertical polarization, the same for $\mathrm{D} 3 \mathrm{H}$ and $\mathrm{D} 3 \mathrm{~V}$. 
Table 1 | Experimental performance under desktop and $1 \mathrm{~km}$ fiber tests

\begin{tabular}{lllllllllll}
\hline $\begin{array}{l}\text { Test } \\
\text { Platform }\end{array}$ & $\begin{array}{l}\text { Average } \\
\text { Photon } \\
\text { Number }\end{array}$ & $\begin{array}{l}\text { Raw } \\
\text { Key } \\
\text { (bits) }\end{array}$ & QBER & $\begin{array}{l}\text { Total } \\
\text { counts }\end{array}$ & $\begin{array}{l}\text { Time } \\
(\mathrm{s})\end{array}$ & $\begin{array}{l}\text { D2 } \\
\text { Counts } \\
\text { Same }\end{array}$ & $\begin{array}{l}\text { D2 } \\
\text { Counts } \\
\text { Diff. }\end{array}$ & $\begin{array}{l}\text { D3 } \\
\text { Counts } \\
\text { Same }\end{array}$ & $\begin{array}{l}\text { D3 } \\
\text { Counts } \\
\text { Diff. }\end{array}$ & $\begin{array}{l}\text { Multiple } \\
\text { (bits) }\end{array}$ \\
\hline Desktop & 0.5 & 185651 & $6.8 \%$ & 4891623 & 3617 & 231482 & 1060186 & 3272114 & 23673 & 8758 \\
Desktop & 0.05 & 7891 & $4.2 \%$ & 151626 & 627 & 9440 & 28386 & 103228 & 319 & 38 \\
$1 \mathrm{~km}$ fiber & 1.0 & 113762 & $5.8 \%$ & 1948287 & 900 & 75628 & 497549 & 1216859 & 7210 & 6376 \\
$1 \mathrm{~km}$ fiber & 0.5 & 11278 & $5.5 \%$ & 191347 & 158 & 7520 & 49224 & 118318 & 487 & 362 \\
\hline
\end{tabular}

Table 1. Performance for counterfactual quantum cryptography system. Table summarizing the various measured parameters characterizing performances for desktop and 1 $\mathrm{km}$ fiber under different average photon number. Besides the symbols introduced in the maintext, here we use "Total Counts" denoting sum of all the detectors D1,D2 and D3. "D2 Counts Same" and "D2 Counts Diff." refer to counting of D2 when Alice and Bob choose the same bit values and different values, respectively. "Multiple" means all the counts for events that there are at lease two detectors clicks. 